\title{
Structural and linear elastic properties of DNA hydrogels by coarse-grained simulation
}

\section{Zhongyang Xing, ${ }^{\dagger}$ Christopher Ness, ${ }^{\ddagger}$ Daan Frenkel, ${ }^{\llbracket}$ and Erika Eiser ${ }^{*, \dagger}$}

†Cavendish Laboratory, University of Cambridge, Cambridge CB3 0HE, United Kingdom

$\ddagger$ Department of Chemical Engineering and Biotechnology, University of Cambridge, Cambridge CB3 0AS, United Kingdom

\Department of Chemistry, University of Cambridge, Cambridge CB2 1EW, United

Kingdom

E-mail: ee247@cam.ac.uk.

\begin{abstract}
We introduce a coarse-grained numerical model that represents a generic DNA hydrogel consisting of Y-shaped building blocks. Each building block comprises three double-stranded DNA arms with single-stranded DNA sticky ends, mimicked by chains of beads and patchy particles, respectively, to allow for an accurate representation of both the basic geometry of the building blocks and the interactions between complementary units. We demonstrate that our coarse-grained model reproduces the correct melting-behaviour between the complementary ends of Y-shapes, and their self-assembly into a percolating network. Structural analysis of this network reveals three-dimensional features consistent with a uniform distribution of inter-building block dihedral angles. When applying an oscillatory shear strain to the percolating system, we show that the system exhibits a linear elastic response when fully connected. We finally discuss to what extent the system's elastic modulus may be controlled by simple changes to the building block complementarity. Our model offers a computationally
\end{abstract}


tractable approach to predicting the structural and mechanical properties of DNA hydrogels made of different types of building blocks.

\section{Introduction}

DNA hydrogels belong to a class of semi-flexible polymeric networks that consist of synthetic nucleotide strands whose binding is governed by base-pair complementarity. ${ }^{1-4}$ The ability to precisely synthesise base sequences, and thus to specify the binding rules a priori, makes such DNA-based systems superior to conventional polymeric networks with non-specific interactions. ${ }^{5,6}$ Thanks to this uniquely programmable self-association, DNA hydrogels have found applications in areas such as drug delivery, ${ }^{1} 3 \mathrm{D}$ cell cultures, ${ }^{7}$ and bio-printing. ${ }^{8}$ In recent works, the phase diagram and some aspects of the rheological behaviour of DNA hydrogels have been reported, ${ }^{4,9-13}$ but robust links between base complementarity and structural and mechanical properties of the resulting hydrogel at the bulk level are missing. Here we establish a simulation model for DNA hydrogels that offers predictions of the structural and mechanical bulk properties of the sample. As we will argue below, our model can help to design and understand experiments, thus providing guidance for future material development.

Computational models of DNA implementing a wide range of coarse-graining levels have been proposed, each focusing on different aspects of the thermodynamics and polymeric nature of DNA. For instance, atomistic models ${ }^{14-16}$ that provide detailed dynamics of nucleotides have advantages in investigating DNA folding and protein-nucleic acid interactions, while bead-spring polymer models with up to 3000 base-pairs represented by one single bead offer a means of obtaining bulk material properties at considerably lower computational expense. ${ }^{17,18}$ Models adopting an intermediate level of coarse-graining, most notably the OxDNA model, ${ }^{19}$ have been utilised in simulating several DNA nanotechnological systems such as molecular machines. ${ }^{20-23}$ In these models ssDNA is presented as a chain of rigid nucleotide beads with effective interacting sites that can reproduce DNA-specific thermody- 
namics and structural properties.

This level of sequence specificity is, however, not always required for simulating selfassembled DNA systems, especially when all possible ssDNA interactions are well known. In these cases, the whole interacting 'sticky' ssDNA sequence at the end of a building block or chain can be treated as a single 'patch' with its potential matching the physical rules ${ }^{24-29}$ from the viewpoint of statistical thermodynamics. This approach maintains the function of ssDNA as a selective bond and at the same time accelerates the simulation process allowing for large numbers of building units that assemble into volume-spanning structures with accurate geometry and topology.

With regard to DNA hydrogels, our interest lies in the structure and mechanics of largescale systems, hence base-pair models ${ }^{19,30-32}$ are too detailed. Computational models of DNA gels self-assembled from branched DNA complexes have been proposed to mimic the bulk behaviour of the system, particularly focusing on the assembly and gelation processes. ${ }^{9,33}$ In those models, structural disorder of such systems has been demonstrated and further discussed, but deeper studies on the possible microstructures of the network, which may facilitate future design of the system, is still absent. Furthermore, the bulk mechanical properties of DNA gels have not been mentioned in any of the present models, but these parameters are actually key in testing some of the functions of the materials. These are limited by the design of the models: in Starr and Sciortino's model, ${ }^{33}$ for instance, the basic geometry of the DNA building blocks is not retained, and thus the microscopic structures cannot be represented accurately. Meanwhile, the OxDNA model and its equivalents ${ }^{9,11}$ are too computationally demanding to be employed for the study of bulk mechanics due to their consideration of the specific base sequences in the building blocks. Therefore, a computational model of DNA hydrogels that both retains the DNA binding rules and is light enough for calculating the bulk properties is in great need.

Here, we discuss one class of DNA hydrogels that is self-assembled from tri-valent building blocks, which are known in the literature as DNA nanostars or Y-shapes in the case of 
A

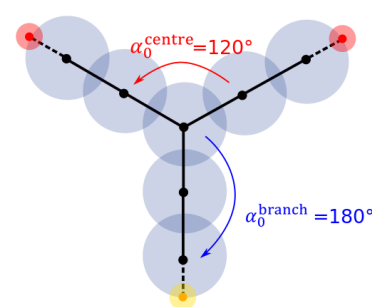

C

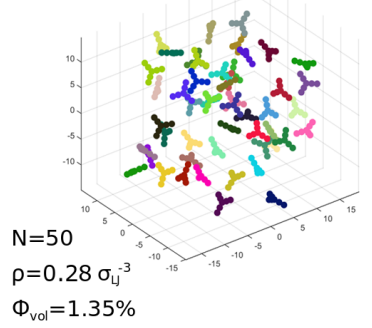

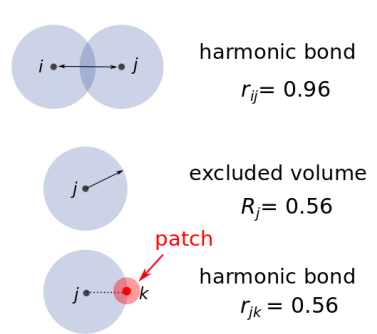

B

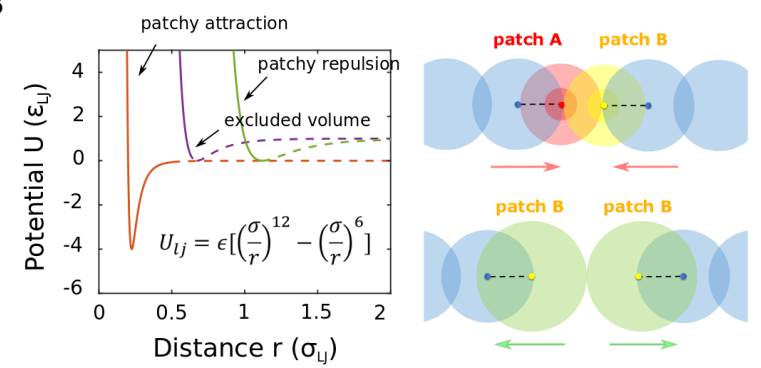

Figure 1: A. Schematic of the bead-spring representation of Y-shaped units. B. (Left) Pairwise potentials used in the model. (Right) Visual description of the designed patchy parameters. In A and $\mathrm{B}$, the large structural beads are represented in light blue, whereas the small patch beads are represented in red and yellow. C. Plots of randomly generated non-overlapping initial configurations at increasing concentrations in a $30 \times 30 \times 30 \sigma_{\mathrm{LJ}}^{3}$ cubic simulation box. The corresponding "per Y-unit" number densities and representative volume fractions are labelled on the bottom left of each panel. 


\section{$2.1 \quad$ Y-shaped building blocks}

The geometric and interaction parameters in our model are chosen to represent the structure of the Y-shaped DNA units used in experimental work conducted by Xing et al. ${ }^{13}$ In our simulation we use a bead-spring model $^{34}$ to represent the dsDNA arms, with attractive patches to mimic the ssDNA sticky ends (Fig.1A,B). All Y-shapes comprise ten beads: one central bead, six structural beads arranged in three arms (colored light blue) and three patch beads (colored red and yellow), one at the end of each arm. The neighbouring beads in each arm are connected by harmonic springs and are kept approximately linear by an angular potential with a minimum at $180^{\circ}$; the three arms are equally distributed around the central bead with position minima at $120^{\circ}$ (see below and Fig. 1A). The three sticky beads (patches) provide attractive sites on the outer surface of the terminal bead of each arm. For simplicity, we define two patch types, patch $A$ and patch $B$, which represent two complementary DNA sequences. The attraction is only enabled for patches of different types, $A-B$.

The model is implemented in LAMMPS, ${ }^{35}$ in which we treat the fundamental units mass $m_{\mathrm{LJ}}$, distance $\sigma_{\mathrm{LJ}}$, energy $\epsilon_{\mathrm{LJ}}$ and the Boltzmann constant $k_{B}$ in reduced units. The reduced time unit is $\tau_{\mathrm{LJ}}=\left(m_{\mathrm{LJ}} \sigma_{\mathrm{LJ}}^{2} / \epsilon_{\mathrm{LJ}}\right)^{1 / 2}$. The subscript 'LJ' stands for Lennard-Jones. Neighbouring beads (and the patches with their host beads) are connected to each other via a harmonic potential

$$
V_{\text {bond }}=K_{\text {bond }}\left(r-r_{0}\right)^{2}
$$

where $r_{0}$ is the equilibrium bond distance and $K_{\text {bond }}$ is the stiffness of the harmonic bond. We set $K_{\text {bond }}$ to $300 \epsilon_{\mathrm{LJ}} / \sigma_{\mathrm{LJ}}^{2}$ throughout, only allowing for small disturbances around the equilibrium distance. ${ }^{34,36}$ We set $r_{0}$ to $0.96 \sigma_{\mathrm{LJ}}$ for bead-bead (solid black lines in Fig $1 \mathrm{~A}$ ) and $0.56 \sigma_{\mathrm{LJ}}$ for the bead-patch bonds (dashed black lines in Fig 1A). The angle constraint is set by the harmonic potential

$$
V_{\text {angle }}=K_{\text {angle }}\left(\alpha-\alpha_{0}\right)^{2},
$$


where $\alpha_{0}$ is the equilibrium angle and $K_{\text {angle }}$ sets the bead/patch chain rigidity. We use $\alpha_{0}^{\text {branch }}$ $=180^{\circ}$ and $\alpha_{0}^{\text {centre }}=120^{\circ}$ to ensure the basic geometric configuration of the Y-shapes, and $K_{\text {angle }}=300 \epsilon_{\mathrm{LJ}} / \mathrm{rad}^{2}$ to constrain the bending of the chains, following Refs. ${ }^{36,37}$

Excluded volumes around the structural beads are introduced using a Weeks-ChandlerAndersen (WCA) potential, which has the same form as the LJ potential but is truncated at its minimum (which occurs at $2^{1 / 6} \sigma \approx 1.12 \sigma$ ):

$$
V_{\mathrm{WCA}}(r, \epsilon, \sigma)= \begin{cases}4 \epsilon\left(\left(\frac{\sigma}{r}\right)^{12}-\left(\frac{\sigma}{r}\right)^{6}\right)+V^{\prime} & r \leq 1.12 \sigma \\ 0 & r>1.12 \sigma\end{cases}
$$

where $\epsilon=\epsilon_{\mathrm{LJ}}$ and $\sigma=\sigma_{\mathrm{LJ}}$, and $V^{\prime}$ is set such that $V_{\mathrm{WCA}}(r=1.12 \sigma)=0$. This potential leads to a repulsive interaction between the structural arms of the Y-shapes, preventing their overlap and crossing. The WCA potential is similarly used provide a short-ranged repulsive interaction for non-complementary patch beads, for which the energy and distance parameters are chosen to be $\epsilon=\epsilon_{\mathrm{LJ}}$ and $\sigma=0.67 \sigma_{\mathrm{LJ}}$, with the cut-off distance and $V^{\prime}$ in Eq. 3 being reset accordingly.

The attraction between complementary patches (here the $A-B$ interaction) follows the same form but is truncated at longer range, leading to a more standard Lennard-Jones potential that has an attractive part:

$$
V_{\mathrm{LJ}}(r, \epsilon, \sigma)=4 \epsilon\left(\left(\frac{\sigma}{r}\right)^{12}-\left(\frac{\sigma}{r}\right)^{6}\right)+V^{\prime \prime}, r \leq r_{\text {cutoff. }} .
$$

Here $\epsilon=4 \epsilon_{\mathrm{LJ}}, \sigma=0.2 \sigma_{\mathrm{LJ}}$ and $V^{\prime \prime}$ are set so that $V_{\mathrm{LJ}}\left(r=r_{\text {cutoff }}\right)=0$. We set $r_{\text {cutoff }}=$ $5 \sigma_{\mathrm{LJ}}$, giving a relatively short-ranged attraction mimicking the ssDNA attractions between complementary sequences. For the case where there are two distinct patch types, namely $A$ and $B$, the combination of WCA and LJ potentials employed in representing the patchy ends of the building-block arms effectively prevents multiple attractions between different ends. For instance, once an $A-B$ pair has successfully formed, the short-ranged WCA 
repulsion between $A-A$ or $B-B$ patch pairs will prevent the formation of a possible three-way binding. Moreover, the steric hinderance offered by the large repulsive structural beads further prevents formation of three-way patch interactions, effectively giving each arm a strict valency of 1, Fig. 1B. Assuming a similar combination of short-ranged attraction and repulsion between complementary and non-complementary patches, the binding behaviour predicted by our model should be agnostic to the precise form of the potential employed. Based on the geometry of an experimental hydrogel, ${ }^{13}$ we take our length scale unit $\sigma_{\mathrm{LJ}}$ to be of order $5 \times 10^{-9} \mathrm{~m}$, while typical bond energies of order $1 \mathrm{kcal} / \mathrm{mol}$ lead to our energy scale unit $\epsilon_{\mathrm{LJ}}$ being of order $7 \times 10^{-21} \mathrm{~J}$. Our unit for $G^{\prime}$ and $G^{\prime \prime}$ referred to later is thus of order $\epsilon_{\mathrm{LJ}} / \sigma_{\mathrm{LJ}}^{3} \approx 10^{4} \mathrm{~Pa}$.

\subsection{Initial configurations}

We first prepare initial configurations by randomly placing non-overlapping Y-shaped units into a cubic simulation box with periodic boundary conditions. Using a Monte Carlo algorithm we placed a seed Y-shape at the centre of the box; then a duplicate Y-shape is generated, given a random rotation and translation, and is labelled as type A or B with equal probability; if the duplication fits in the simulation box and does not overlap with the existing units, it is retained, otherwise, the duplication is abandoned; this procedure is run until the number of desired Y-shapes meets a pre-set number density. The resulting configuration is then used as the configurational input for the following simulation. Fig. 1C shows examples of initial configurations for different number denisties. The data presented hereafter represent ensemble averages of 10 realisations.

The cubic simulation box has length $30 \sigma_{\mathrm{LJ}}$, and the number density is set by varying the number of the $Y$-shaped units therein. We define a per-Y-shape volume $V_{\mathrm{Y}}=\left(\pi r^{2} \times l\right) \times 3 \approx$ $7.3 \sigma_{\mathrm{LJ}}^{3}$, with $r=0.56 \sigma_{\mathrm{LJ}}$, and $l=2.48 \sigma_{\mathrm{LJ}}$, and characterise the concentration based on the approximate volume fraction $\phi_{\mathrm{vol}}$ and the number density $\rho$. 


\subsection{Simulation details}
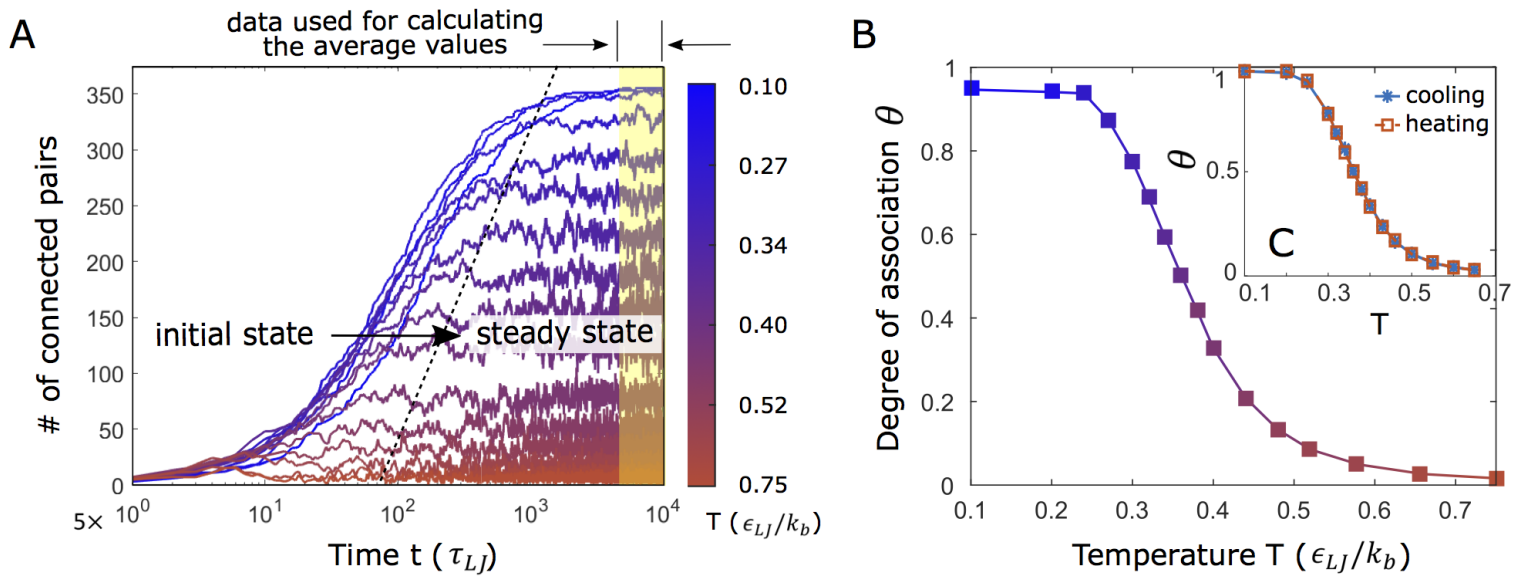

Figure 2: A. Illustration of equilibration process quantified by the number of connected pairs for temperatures between $0.1 \rightarrow 0.75 \epsilon_{\mathrm{LJ}} / k_{B}$. The system starts at a randomly generated non-overlapping initial state (Fig.1C) and eventually reaches its steady state, where the number of connected pairs reaches a plateau. B. Degree of association $\theta(T)$ calculated from the averaged number of connected pairs in Fig.2A (masked in yellow). C. Hysteresis test for cooling-down and heating-up ramps. All the data are taken on a system with $\rho=1.39 \sigma_{\mathrm{LJ}}^{-3}$ in the simulation box $=30 \times 30 \times 30 \sigma_{\mathrm{LJ}}^{3}$.

We perform all coarse-grained molecular dynamics simulations using Langevin dynamics, in which the trajectories of each bead are computed according to

$$
m \frac{d^{2} \mathbf{x}}{d t^{2}}=-\nabla U(\mathbf{x})-\lambda \frac{d \mathbf{x}}{d t}+\eta(t)
$$

where $\mathbf{x}$ and $m$ (in units $m_{\mathrm{LJ}}$ as above) are the position and mass of a single bead respectively. $U(\mathbf{x})$ is the bead interaction potential (that is, the sum of the relevant $V$ terms), the damping parameter $\lambda$ is large to approximate over-damped conditions, and $\eta(t)$ is a noise term from interactions with a stochastic heat bath via random forces and dissipative forces. $\eta(t)$ can be written as $\eta(t)=\sqrt{2 \gamma k_{B} T} R(t)$, with the temperature $T$ ranging from $0.05 \epsilon_{\mathrm{LJ}} / k_{B}$ to $0.7 \epsilon_{\mathrm{LJ}} / k_{B}$ for all the simulations, and $R(t)$ is a delta-correlated stationary Gaussian process with zero-mean. We first equilibrate the system at a fixed temperature $T_{\mathrm{i}}\left(0.05 \epsilon_{\mathrm{LJ}} / k_{B} \leq\right.$ $\left.T_{\mathrm{i}} \leq 0.7 \epsilon_{\mathrm{LJ}} / k_{B}\right)$, starting from the initial configurations aforementioned. The numerical 
time step was set to $0.005 \tau_{\mathrm{LJ}}$, and each of the simulations runs for $5 \times 10^{4} \tau_{\mathrm{LJ}}$ to ensure that equilibrium is reached. We use the number of connected patchy pairs to characterize the connectivity of the network, shown in Fig.2A. This quantity increases during equilibration, reaching a plateau whose value depends on $T$ and the number density $\rho$. We use the degree of association $\theta$ to evaluate the connectivity of the network, which we define here as

$$
\theta=\frac{M}{\left(Q_{\text {valence }} \times N\right) / 2} .
$$

Here $M$ is the number of connected patchy pairs, $N$ is the total number of Y-shaped units and $Q_{\text {valence }}$ is the building-block valency, which is 3 in our model by construction. As the denominator represents the maximum number of connected patchy pairs for a system of $N$ units, $\theta$ varies between 0 at high temperatures, where we have a gas of Y-shapes, and 1 at very low temperatures, when all possible bonds in the system are formed. As shown in Fig. 2A, we compute values of $M$ time-averaged over the steady state period. Fig. 2B plots $\theta$ against temperature $T$, which we refer to hereafter as the melting curve. A hysteresis test is run to confirm that the systems stay in equilibrium. To do so we first take the equilibrated system at temperature $T=0.65 \epsilon_{\mathrm{LJ}} / k_{B}$ as the input configuration, and then cool it down to $T=0.6 \epsilon_{\mathrm{LJ}} / k_{B}$ until another steady state is reached. Likewise, the final steady state of the $(i-1)^{t h}$ step was chosen to be the starting point of the $i^{\text {th }}$ step, whose steady state serves as the starting state for step $i+1$, and so forth. Such a slow cooling or heating cycle is similar to hybridization cycles probed by standard UV-vis spectroscopy measurements, which are used to determine the melting behaviour of a given DNA duplex. ${ }^{38,39}$ Fig. $2 \mathrm{C}$ shows cooling and heating ramps for temperatures between $0.1 \epsilon_{\mathrm{LJ}} / k_{B}$ and $0.65 \epsilon_{\mathrm{LJ}} / k_{B}$. No hysteresis is observed, demonstrating that we capture the system's equilibrium hybridization behaviour. 


\section{DNA thermodynamics}

Thermodynamic hybridization for short-stranded DNA can be described by a two-state model. We first assume that an equimolar mixture of complementary ssDNA (molecules are noted as A and B) can hybridize into dsDNA (molecules noted as AB). For ideal mixtures, the equilibrium constant $K_{a}$ for this reaction can be written as

$$
K_{a}=\frac{[A B] /[\varnothing]}{([A] /[\varnothing])([B] /[\varnothing])}=\exp \left(-\beta \Delta G^{\varnothing}\right),
$$

where $[\mathrm{A}],[\mathrm{B}]$ and $[\mathrm{AB}]$ refer to the concentration of ssDNA A, ssDNA B and dsDNA AB, respectively. $[\varnothing]$ is the standard molar state concentration and $\beta=1 / k_{B} T$, where $k_{B}$ is the Boltzmann constant, and $T$ is the temperature in Kelvin. $\Delta G^{\varnothing}$ is the standard Gibbs energy for DNA hybridization, which can be estimated using the SantaLucia thermodynamic model. ${ }^{40}$ We note that Eq. 7 strictly applies only for reaction $A+B \rightleftharpoons A B$, where $\mathrm{A}, \mathrm{B}$ and $\mathrm{AB}$ are free in solution at low densities, but we find it a useful approximation for our data at moderate concentrations and temperatures. Many models have been proposed to predict the phase behaviour of associating fluids. ${ }^{41}$

To transfer these values into simulation parameters, we furthermore write the DNA concentration in terms of a number density, and therefore the concentrations of $\mathrm{A}, \mathrm{B}$ and $\mathrm{AB}$ are denoted as $\rho_{\mathrm{A}}, \rho_{\mathrm{B}}$, and $\rho_{\mathrm{AB}}$, respectively. Starting with the fully disassociated state $\theta=0$, and with an equal mixture of $\mathrm{A}$ and $\mathrm{B}$, we write down the initial number densities as

$$
\rho_{\mathrm{A}}^{\circ}=\rho_{\mathrm{B}}^{\circ}=\rho,
$$

so that at a given temperature $T$ the fraction of basepairs (bonds) formed can be related to 
the number densities of $\mathrm{AB}, \mathrm{A}$ and $\mathrm{B}$ via

$$
\rho_{\mathrm{AB}}=\rho \theta
$$

$$
\rho_{\mathrm{A}}=\rho_{\mathrm{B}}=\rho(1-\theta) .
$$

The left-hand term in Eq. 7 becomes

$$
K_{a}=\frac{\rho_{\mathrm{AB}} \rho^{\varnothing}}{\rho_{\mathrm{A}} \rho_{\mathrm{B}}}=\frac{\theta \rho^{\varnothing}}{\rho(1-\theta)^{2}},
$$

with $\rho^{\varnothing}=6.022 \times 10^{26} \mathrm{~m}^{-3}$ being the standard number density. At fixed total volume $V$ and number of building blocks $N$, the number density can be written as $\rho=N / V$. Hence, Eq. 10 can be expressed as

$$
K_{a}=\frac{\theta}{N(1-\theta)^{2}} \times \rho^{\varnothing} V .
$$

Substituting $K_{a}$ with Eq. 11, Eq. 7 can be rewritten as

$$
\frac{\theta}{N(1-\theta)^{2}} \times \rho^{\varnothing} V=\exp \left(-\beta \Delta G^{\varnothing}\right)
$$

where the left-hand side purely relates to the number density and the degree of association, while the right-hand term is associated with the Gibbs free energy of binding. Hence we can redefine the reaction constant as

$$
K_{a}^{*}=\frac{\theta}{N(1-\theta)^{2}},
$$

and write Eq. 12 as

$$
K_{a}^{*}=\exp \left(-\beta \Delta G^{\varnothing}\right) / \rho^{\varnothing} V .
$$

For a given ssDNA sequence, $\Delta G^{\varnothing}$ is constant at constant temperature. ${ }^{40}$ Therefore, at fixed temperature $T$ and volume $V, K_{a}^{*}$ should remain the same value at equilibrium regardless of the initial state. Note, however, that at higher concentrations Eqn. 13 may break down as the activity coefficients of the various species may differ from unity. Thus we can test the validity 
of our simulation model by calculating Eq. 13 for varying $N$ and $\theta$. Further, combining Eqs 13 and 14 we can compute the melting temperature $T_{m}=-\Delta G^{\varnothing}\left(k_{B} \ln \left(\rho / 2 \rho^{\varnothing}\right)\right)^{-1}$, which is defined as the temperature at which half of all possible bonds are formed $(\theta=1 / 2)$.
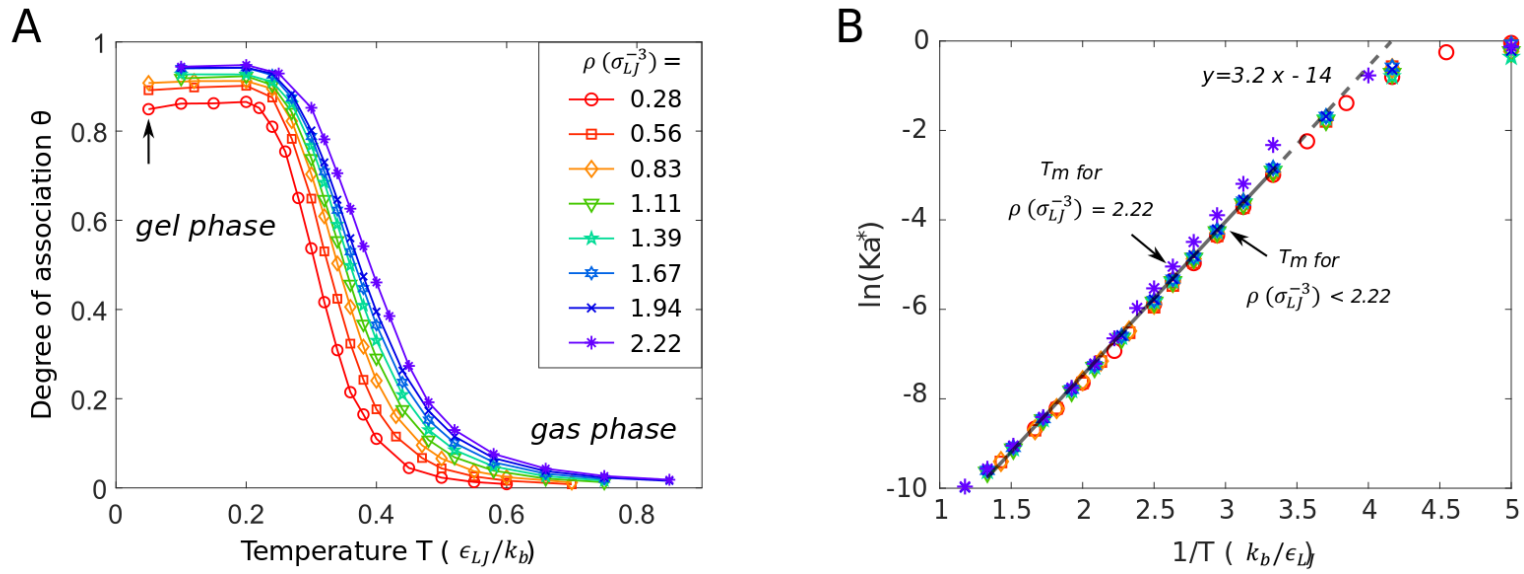

Figure 3: A. The simulation-determined melting curves for samples at number density $\rho=0.28 \rightarrow 2.22 \sigma_{\mathrm{LJ}}^{-3}$. B. Arrhenius plot obtained from $\theta(T)$ as described by Eq.15.

Fig. 3A shows melting curves for systems with number densities $\rho$ varying from 0.28 to $2.22 \sigma_{\mathrm{LJ}}^{-3}$. It is important to note that these densities have been chosen such that the system does not undergo a macroscopic phase separation into a DNA-rich gel and a DNApoor liquid phase but rather is brought continuously from the liquid to an equilibrium gel phase when cooled down, as observed in various experimental works. ${ }^{4,10,13}$ Again, each data point $\theta(\rho, T)$ is averaged over 10 independent realisations following the equilibrating strategy described above. As expected, $\theta \rightarrow 0$ at high temperatures for all concentrations, indicating that all patchy pairs are dissociated and the system is in a gaseous phase of $\mathrm{Y}$-shapes. At temperatures well below the system's $T_{m}, \theta$ tends towards 1 , reflecting the fact that almost all possible bonds have formed rendering the system a percolating gel. We also observe that $T_{m}$ shifts towards higher temperatures, demonstrating that our simulation model captures the concentration dependence of $T_{m}$.

Another interesting observation following from Figs. 2 and $3 \mathrm{~A}$ is that $\theta$ never reaches 1 even at $T \rightarrow 0$, meaning that not all the patches are bonded even well below $T_{m}$. This is in contrast to the thermodynamics of DNA hybridization in which all complementary 
ssDNA oligomers will bind to each other forming non-connected duplexes. ${ }^{38}$ We argue that this behaviour is purely due to geometric restrictions imposed by the angular potentials keeping the three arms in the Y-shapes at a rather rigid angle of $120^{\circ}$ and in plane. This is a rather realistic representation as we know that dsDNA has a much longer persistence length than the arm length used in experiments ${ }^{13}$ and an observation that we confirmed in simulations with the more detailed OxDNA model. However, our model permits free rotation between two Y-shapes when bonded, thus allowing to form a 3D network with these flat structures. Furthermore, two bonded arms are not completely stiff but can form at an angle, which is controlled by the excluded volume of the outer beads and the patch size, reflecting the flexible linkers made of non-binding thymine bases built into our experimental realization. ${ }^{13}$ Hence when the network starts to form, some bonds will be physically not close enough to hybridize, as the rest of the Y-shapes are connecting to others in the network. This topological hindrance will lead to $\theta$ not reaching full association. Moreover, one would expect the low-temperature plateau to decrease even more for lower concentrations as is demonstrated in Fig. 3. The latter also explains the slight increase of $\theta$ in the $\rho=0.28 \sigma_{\mathrm{LJ}}^{-3}$ curve when heating from $T=0.1$. At these low temperatures thermal fluctuations are not sufficient to locally break a strained bond to rearrange into a lower free energy configuration. When the temperature goes up slightly, these strained bonds can dissociate and form new pairs bringing the system closer to its thermodynamic equilibrium state.

From the melting curves we can also extract the binding enthalpy by rewriting Eq. 14 in terms of the enthalpy $\Delta H$ and entropy $\Delta S$ of binding:

$$
\ln K_{a}^{*}=-\left(\frac{\Delta H}{k_{B} T}+\Delta S\right) / \rho^{\varnothing} V
$$

Recasting our melting data in the form of an Arrhenius plot, Fig.3B, we observe rather weak concentration dependence of the rate constant at all temperatures consistent with prior results. ${ }^{40}$ The relationship $\ln \left(K_{a}^{*}\right) \propto 1 / T$ computed from our simulation data holds for 
temperatures above $T_{m}$ and leads to $-\frac{\Delta H}{k_{B} \rho^{\varnothing} V} \approx 3.2$. Below this temperature, many-body association and structural effects lead to deviations from the theory in Eq 15, as expected since the linear prediction ${ }^{40}$ only applies strictly to hybridization of DNA strands forming linear duplexes that are free in solution. Nonetheless, these results further demonstrate that our coarse-grained approach is a good model for representing tenuous DNA-hydrogels of nano-stars.

\section{Structural properties}

The structural analysis of our DNA hydrogels is guided by the radial distribution function $g(r)$ of the central beads of the Y-shapes, which is given by

$$
g(r)=\frac{1}{4 \pi r^{2} \rho N} \sum_{i=1}^{N} \sum_{j \neq i}^{N}\left\langle\delta\left(\left|r_{i j}-r\right|\right)\right\rangle .
$$

Here $N$ is the total number of the central beads, and $\rho$ is the averaged number density of the central beads across the whole system. The sum counts the total number of the central bead pairs at the distance $r$. We average this quantity over all equilibrium configurations.

Fig. 4A shows a schematic of the geometry of four Y-shaped units connected in a chain. They are marked in two different colours (green and blue) indicating units with different patch types (patch A and patch B). We define the centre points of the central beads (darker green or darker blue) in a row as points $A_{1}, A_{2}, A_{3}$ and $A_{4}$, and the planes where the corresponding $\mathrm{Y}$-shaped units stay on as planes $P_{1}, P_{2}, P_{3}$ and $P_{4}$. The sketch follows the assumptions below:

1. All beads of one given Y-shaped unit lie in the same plane.

2. The arms of two connected patches are aligned along their principal axis. Fig.4A(a) shows the case that they are not aligned. 
A

(a)

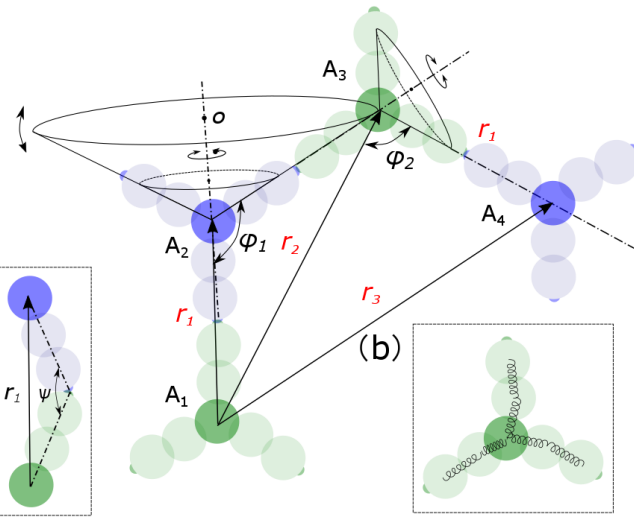

B low $T$ high T

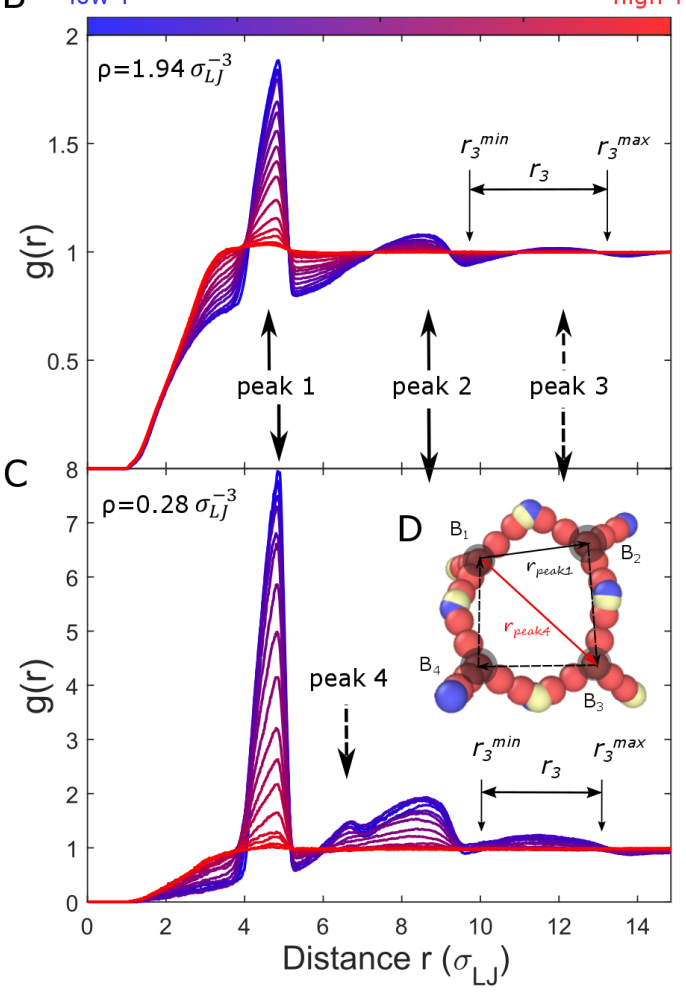

Figure 4: A. Schematic of the typical configuration of four Y-shaped units associated in a row, drawn in blue and green respectively to indicate different patchy types. $A_{1}, A_{2}$, $A_{3}$ and $A_{4}$ denote the centre point of the centre beads (darker color). B-C. Central-bead radial distribution function $\mathrm{g}(\mathrm{r})$ for $\rho=1.94$ and $0.28 \sigma_{\mathrm{LJ}}^{-3}$ systems. D. Snapshot of the ring structure for four Y-shaped units. 
3. The three beads in sequence in the same arm are properly aligned. Fig.4A(b) shows the case where they are unaligned.

4. The plane $P_{i+1}$ can rotate freely around the $\overline{A_{i} A_{i+1}}$ axis. We assume the rotation, or dihedral angle of plane $P_{i}$ and $P_{i+1}$, follows a uniform distribution.

Our choice of $K_{\text {bond }}$ and $K_{\text {angle }}$ ensure that assumptions 1-3 are satisfied to a good approximation. According to these assumptions, we estimate the three typical lengths marked as $r_{1}, r_{2}$ and $r_{3}$, which represent the distance of $\overline{A_{1} A_{2}}, \overline{A_{2} A_{3}}$ and $\overline{A_{3} A_{4}}$ respectively, as well as angles $\varphi_{1}$ and $\varphi_{2}$ that represent $\angle A_{1} A_{2} A_{3}$ and $\angle A_{1} A_{3} A_{4}$ (see Fig. $4 \mathrm{~A}$ ). We calculate that $r_{1}=4.96 \sigma_{\mathrm{LJ}}$, and $r_{2}=8.59 \sigma_{\mathrm{LJ}}$ with $\varphi_{1}$ at a fixed value of $120^{\circ}$. Though $\varphi_{2}$ is unknown, if we take the uniform distribution for the rotation angle, the expectation value of $r_{3}$ can be calculated as $\approx 2.37 r_{2}$, corresponding to $11.5 \sigma_{\mathrm{LJ}}$. Details of the calculation are given in the Supporting Information S1.

We measured the radial distribution function $g(r)$ of the central beads of the systems at various concentrations and temperatures, at the equilibrium states acquired before. All measurements were time-averaged over $10^{6}$ configurations from the time series and then over 10 independent realisations at given $(N, T)$, with $T$ chosen to cover the full melting region. Fig. $4 \mathrm{~B}$ shows the radial distribution function at $\rho=1.94 \sigma_{\mathrm{LJ}}^{-3}$ for across a range of $T$. For all the measurements, $g(r)=0$ at $r<1.12 \sigma_{\mathrm{LJ}}$, which is the cut-off distance of the WCA potential applied to the central beads. At high temperature, $g(r)$ is approximately flat, showing a gaseous phase consistent with the melting temperature results. At low temperature, we obtain three peaks at distance $r_{p 1}=4.85 \sigma_{\mathrm{LJ}}, r_{p 2}=8.60 \sigma_{\mathrm{LJ}}$ and $r_{p 3}=11.84 \sigma_{\mathrm{LJ}}$. This is in good agreement with our theoretical prediction where $\left\langle r_{1}\right\rangle=4.96 \sigma_{\mathrm{LJ}},\left\langle r_{2}\right\rangle=8.59 \sigma_{\mathrm{LJ}}$ and $\left\langle r_{3}\right\rangle=11.76 \sigma_{\mathrm{LJ}}$, demonstrating that in the bulk structure, the distribution of dihedral angles is roughly uniform. The minor off-set in each of the peaks is mainly due to misalignments that violate our above assumptions, as shown in Fig.4A(a) and (b). Accordingly, the corresponding value of $\varphi_{2}$ is calculated as $120.6^{\circ}$ for $r_{3}=11.76 \sigma_{\mathrm{LJ}}$ as above (see Eq. A3 ). This shows that the basic configuration of connected Y-shaped units simply do not lie in a 
plane $\left(\varphi_{2}=90^{\circ}\right.$ or $\left.150^{\circ}\right)$, which furthermore illustrates that the system prefers to form a $3 \mathrm{D}$ network instead of a $2 \mathrm{D}$ sheet.

The results of the system at low concentration $\left(\rho=0.28 \sigma_{\mathrm{LJ}}^{-3}\right.$, Fig. $\left.4 \mathrm{C}\right)$ show an unexpected 4th peak between $r_{1}$ and $r_{2}$. As discussed in detail in Supporting Information S1, $r_{1}$ is the shortest characteristic length in the system, and, assuming the planar arrangement in Fig $4 \mathrm{~A}, r_{2}$ is the second shortest. We find that at low concentrations there is a preferred closed ring structure formed by 4 Y-shaped units (Fig. 4D) rather than an open linear structure (Fig. 4A). The diagonal distance $\overline{B_{1} B_{3}}$ in the ring structure indeed matches the value of $r_{p 4}$. The ring structure results from considerable bending from the patchy connection point between to associated arms, and is only observed for the very dilute case, where it is widespread. In order to form a 4 -membered ring, $l_{1}$ deviates from its most probable length, costing extra bending energy; but the gain in enthalpy by connected patchy pairs in the ring structure compensates this bending-energy penalty. Supporting Information S3 presents the radial distribution functions of the systems in the absence of shearing. We can clearly see that peak 4 is only present for $\rho=0.28$ and $0.56 \sigma_{\mathrm{LJ}}^{-3}$, and at higher concentration we can only observe 3 typical peaks. This indicates that at high concentrations equal to and above $\rho=0.83 \sigma_{\mathrm{LJ}}^{-3}$, the system is densely packed, which frustrates ring formation; and at low concentrations there is still some empty space, so some free patches eventually detect close by counterparts to bind and thus lower the systems' binding Gibbs free energy. The structural information our model can serve as reference for more complicated designs using for example DNA nanostars in which each sticky end has a different binding energy. Hence, strict connecting rules can be used to explore the possibility to form more hierarchical open networks. 
A

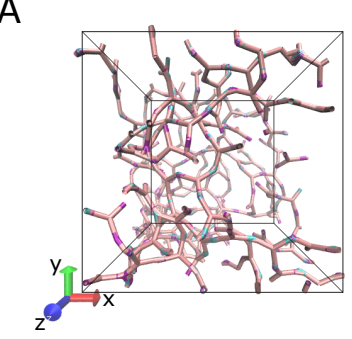

C

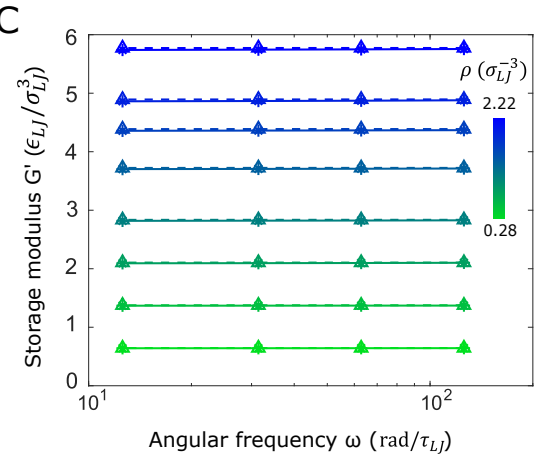

\section{$5 \quad$ Response to oscillatory shear}

We explored the rheological properties of the hydrogels by applying a shear flow (with flow in $x$ and gradient in $y$ ) to the networks formed after equilibration (see Fig. 5A) and subsequent cooling to $T=10^{-7} \epsilon_{\mathrm{LJ}} / k_{B}$, such that thermal motion may be neglected. This approach gives a simplified prediction of how the overall structure responds elastically to mechanical perturbations; in future work we will explore the rheology near to the melting transition.

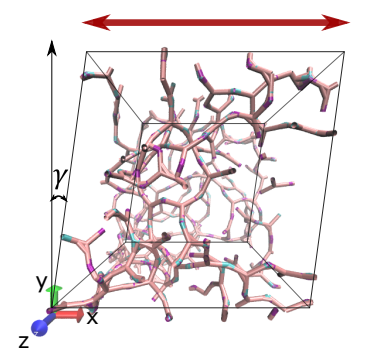

D

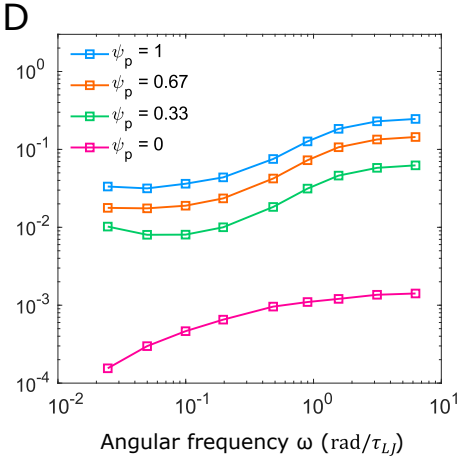

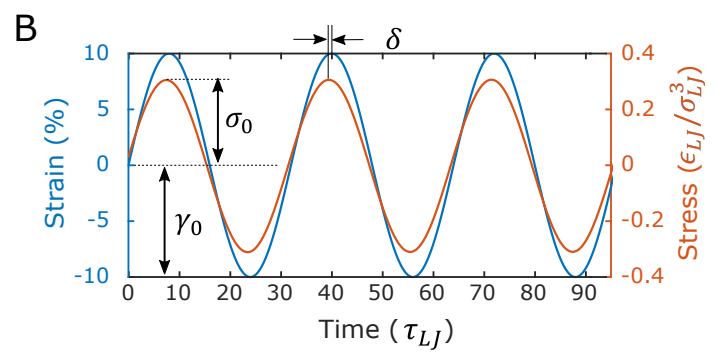

$\mathrm{E}_{10}$

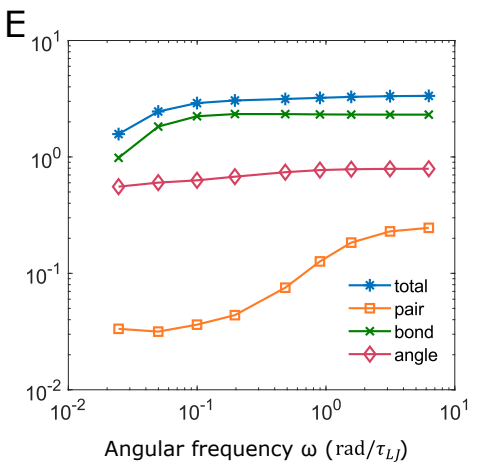

Figure 5: A. Snapshots of the fully associated system $(\theta \rightarrow 1)$ in the unsheared (left) and sheared state (right). B. Illustration of strain and stress time series for three cycles. C. Plot of storage modulus $G^{\prime}(\omega)$ at strain amplitudes of $10 \%$ (solid lines with star markers) and $1 \%$ (dashed lines with triangle markers) respectively. The color scale represents the number density of the system. D. Storage modulus $G^{\prime}(\omega)$ with active patches at $1,0.67,0.33$ and 0 $\left(\gamma_{0}=10 \%\right)$. E. Separated pair, bond and angle contributions and the overall stress to the storage modulus $G^{\prime}(\omega)\left(\gamma_{0}=10 \%\right)$.

A Nose-Hoover temperature thermostat accompanied with the SLLOD equation of motion is employed, ensuring that the simulation is carried out at fixed volume $V$ and temperature $T$. Using the SLLOD equation of motion, we subtract the streaming velocity of the particles caused by the re-shaping of the simulation box while shearing. ${ }^{42,43}$ 
The positions and velocities of the particles are re-mapped every $0.001 \tau_{\mathrm{LJ}}$ for transient shear, and $0.0002 \tau_{\mathrm{LJ}}$ for oscillatory flow to eliminate integration errors in the latter case (Fig. S4, Supporting Information S3). To achieve oscillatory shear, we impose a timedependent strain and rate of the strain given by Eq. 17

$$
\gamma(t)=\gamma_{0} \sin (\omega t),
$$

$$
\dot{\gamma}(t)=\gamma_{0} \omega \cos (\omega t),
$$

where $\gamma_{0}$ is the amplitude of the shear deformation, and $\omega$ is the shearing frequency. Assuming that we remain in the linear viscoelastic regime, the resulting shear stress (in the $x y$ direction) can be written as:

$$
\sigma(t)=\sigma_{0} \sin (\omega t+\delta)
$$

Here $\sigma_{0}$ is the amplitude of the shear stress, and $\delta$ is the phase shift of the stress response due to the different elastic and viscous contributions of the material at various frequencies. The storage $G^{\prime}(\omega)$ modulus can be obtained according to Eq. 19:

$$
G^{\prime}=\frac{\sigma_{0}}{\gamma_{0}} \sin (\delta)
$$

We first apply an oscillatory shear deformation with amplitude $\gamma_{0}$ at angular frequency $\omega$, acquiring time-series of the resulting shear stress $\sigma(t)$ from the simulation. The measured shear stress $\sigma(t)$ shows a sinusoidal shape with a phase shift $\delta$ from the input shear strain as expected. A typically measured stress response is illustrated in Fig. 5B. We average $\sigma(t)$ for every 3 cycles and then compute $\sigma_{0}$ and $\delta$ (Eq. 18), before using Eq. 19 to obtain $G^{\prime}$. Results for the storage modulus $G^{\prime}(\omega)$ are presented in Figure $5 \mathrm{C}$, for $\gamma_{0}=0.01$ and $\gamma_{0}=0.1$ across a range of number densities. We also calculated the viscoelastic properties using the stress autocorrelation function as an input to the Green-Kubo relation, which closely matches that obtained by mechanical spectroscopy (see Supporting Information S4).

Our results show that $G^{\prime}(\omega)$ increases with the system density, which is not surprising 
because the elasticity is related to the number of harmonic bonds in the system. Hence the more connected Y-shapes are present in the system, the higher is the elasticity. We can clearly see that at fixed angular frequency $\omega, G^{\prime}(\omega)$ increases roughly linearly with the density of the system as expected. For frequencies large relative to the characteristic timescale of the simulation (that is, $\omega>1 \mathrm{rad} / \tau_{\mathrm{LJ}}$ ), we find a small phase angle throughout, indicative of a linear elastic response irrespective of the straining amplitude. For smaller frequencies we find that $G^{\prime}(\omega)$ drops off, indicating that structural relaxation leads to dissipation and thus a viscous contribution to the rheology. This drop off occurs at an approximately fixed value of the characteristic shear rate $\gamma_{0} \omega$. Indeed, under linear strain ramps (that is, $\gamma(t)=\dot{\gamma} t$, see Fig. S3 in Supporting Information S4) the stress increase is linear in strain while $\dot{\gamma}=\tau_{\mathrm{LJ}}^{-1}$, and becomes sub-linear for smaller shear rates. In the following, we report results measured using $\gamma_{0}=0.1$, which gives significantly better statistics and a wider range of accessible angular frequencies.

Fig. 5E shows shear moduli calculated based on different potential contributions (pair, bond and angle) at number density $\rho=1.4 \sigma_{\mathrm{LJ}}^{-3}$. Other than at extremely low frequency, the contributions $G_{\text {angle }}^{\prime}$ and $G_{\text {bond }}^{\prime}$ are nearly independent of frequency $\omega$, which is in line with expectation as the potentials are harmonic. In contrast, the $G_{\text {pair }}^{\prime}$ is increasing with frequency $\omega$, meaning the pair interactions become more important over short time scales. Since a single Y-shaped unit is composed of ten beads linked by bonds and shaped by angle constraints, it is not surprising that $G_{\text {pair }}^{\prime}$ from patchy interactions only show lower values than $G_{\text {bond }}^{\prime}$ and $G_{\text {angle }}^{\prime}$.

We finally demonstrate the control over the material's rheological properties that we can exert by tuning the patchy interactions. As an illustrative example, we modify the complementarity simply by 'switching off' some of the patches at random (i.e. by switching them to being universally repulsive, see Figure 1B): thus, the proportion of patches that remain active is $\Psi_{p}$. As shown in Fig. $5 \mathrm{D}, G_{\text {pair }}^{\prime}$ shifts progressively downward as patches are deactivated. It is clearly seen that while there are still patchy particles present, the frequency- 
dependent elastic modulus response resembles the same shape as when fully connected, presenting a plateau at low frequencies, which shows a network feature. However, when all the patchy interactions are disabled, the low-frequency plateau disappears at low frequencies, due to the loss the connectivity of the bulk system. It also indicates that if we change the binding rules slightly, we can achieve good control over the pair contribution to $\sigma(t)$. This shows that the chemistry of DNA hydrogels allows us to manipulate their rheology in a way that is not possible (or at least more difficult) with conventional polymeric materials.

\section{Conclusion}

We have introduced a coarse-grained model for a binary DNA hydrogel system, made of rigid double-stranded Y-shaped DNA nanostars with patchy ends. We demonstrated that the melting behaviour of the model matches a simplified DNA thermodynamic theory well. We also studied the structural properties predicted by the model at various concentrations and temperatures, proving the three dimensional structural information of the system. We find that the network structure largely conforms to a uniform distribution of dihedral angles. Interestingly, at low temperatures we find locally higher order in the form of ring structures, which is difficult to verify experimentally. Conducting transient and oscillatory rheological studies we also gained furtehr insight into the relation between the macroscopic elasticity of such a DNA-hydrogel and the local structure. The storage modulus results show the elasticity of the system when fully connected, but due to the zero-temperature condition, all the frictional interactions are switched off and the loss modulus is actually not reliable (therefore do not not show it), demonstrating the limitation of the model, which we hope to resolve in the future. For example, we could calculate the centre-of-mass stress instead of per-atom stress, and the former should be able to screen out the intra-molecular interactions and therefore amplify the inter-molecular interactions mainly caused by the ssDNA sticky ends (represented as patchy interaction here). Nevertheless, our coarse-grained model is 
robust enough to capture the overall systems network formation and scaling of the elasticity with the number of bonds per volume. Moreover, it gives better insight into local structural arrangements that cannot be directly tested in experiments.

Furthermore, our model is computationally efficient compared to current DNA simulation models ${ }^{30-32}$ as we neglect the binding specificity using a patch interactions with given interaction strength. This is preferable for predicting the structural and dynamic properties of larger scale DNA self-assembly systems with repeating building blocks, which is normally very costly if the per-nucleotide interaction is considered. The key parameters for the building blocks (i.e. angles, bonds, geometry, etc.) can be optimised by matching with lower-level coarse-grain simulations such as OxDNA model provide quantitative predicts for real materials. Hence, this model can be used in the design of DNA networks with more interesting viscoelastic properties in the future.

\section{Acknowledgement}

ZX receives financial support from National University of Defense Technology, China. CN acknowledges the Maudslay-Butler Research Fellowship at Pembroke College, Cambridge for financial support. ZX and CN contributed equally. We thank Jiangbin Zhang for writing the Matlab script of randomly generated initial configurations, Clara Wanjura for performing the spatial distribution calculation, and Aleks Reinhardt for useful discussion on patchy particles and DNA melting.

\section{References}

(1) Um, S. H.; Lee, J. B.; Park, N.; Kwon, S. Y.; Umbach, C. C.; Luo, D. Enzyme-catalysed assembly of DNA hydrogel. Nature materials 2006, 5, 797.

(2) Lee, J. B.; Peng, S.; Yang, D.; Roh, Y. H.; Funabashi, H.; Park, N.; Rice, E. J.; Chen, L.; 
Long, R.; Wu, M.; Dan, L. A mechanical metamaterial made from a DNA hydrogel. Nature Nanotechnology 2012, 7, 816.

(3) Cheng, E.; Xing, Y.; Chen, P.; Yang, Y.; Sun, Y.; Zhou, D.; Xu, L.; Fan, Q.; Liu, D. A pH-triggered, fast-responding DNA hydrogel. Angewandte Chemie 2009, 121, 77967799.

(4) Biffi, S.; Cerbino, R.; Bomboi, F.; Paraboschi, E. M.; Asselta, R.; Sciortino, F.; Bellini, T. Phase behavior and critical activated dynamics of limited-valence DNA nanostars. Proceedings of the National Academy of Sciences 2013, 110, 15633-15637.

(5) Jones, M. R.; Seeman, N. C.; Mirkin, C. A. Programmable materials and the nature of the DNA bond. Science 2015, 347, 1260901.

(6) Wang, D.; Hu, Y.; Liu, P.; Luo, D. Bioresponsive DNA Hydrogels: Beyond the Conventional Stimuli Responsiveness. Accounts of Chemical Research 2017, 50, 733-739.

(7) Lee, J. B.; Roh, Y. H.; Um, S. H.; Funabashi, H.; Cheng, W.; Cha, J. J.; Kiatwuthinon, P.; Muller, D. A.; Luo, D. Multifunctional nanoarchitectures from DNA-based ABC monomers. Nature Nanotechnology 2009, 4, 430.

(8) Li, C.; Faulkner-Jones, A.; Dun, A. R.; Jin, J.; Chen, P.; Xing, Y.; Yang, Z.; Li, Z.; Shu, W.; Liu, D.; Duncan, R. Rapid formation of a supramolecular polypeptide-DNA hydrogel for in situ three-dimensional multilayer bioprinting. Angewandte Chemie International Edition 2015, 54, 3957-3961.

(9) Rovigatti, L.; Smallenburg, F.; Romano, F.; Sciortino, F. Gels of DNA nanostars never crystallize. ACS Nano 2014, 8, 3567-3574.

(10) Biffi, S.; Cerbino, R.; Nava, G.; Bomboi, F.; Sciortino, F.; Bellini, T. Equilibrium gels of low-valence DNA nanostars: a colloidal model for strong glass formers. Soft Matter 2015, 11, 3132-3138. 
(11) Rovigatti, L.; Bomboi, F.; Sciortino, F. Accurate phase diagram of tetravalent DNA nanostars. The Journal of Chemical Physics 2014, 140, 154903.

(12) Fernandez-Castanon, J.; Bomboi, F.; Rovigatti, L.; Zanatta, M.; Paciaroni, A.; Comez, L.; Porcar, L.; Jafta, C. J.; Fadda, G. C.; Bellini, T.; Sciortino, F. Small-angle neutron scattering and molecular dynamics structural study of gelling DNA nanostars. The Journal of Chemical Physics 2016, 145, 084910.

(13) Xing, Z.; Caciagli, A.; Cao, T.; Stoev, I.; Zupkauskas, M.; O’Neill, T.; Wenzel, T.; Lamboll, R.; Liu, D.; Eiser, E. Microrheology of DNA hydrogels. Proceedings of the National Academy of Sciences 2018, 115, 8137-8142.

(14) Cheatham, T. E. Simulation and modeling of nucleic acid structure, dynamics and interactions. Current Opinion in Structural Biology 2004, 14, 360-367.

(15) Fadrna, E.; Spackova, N.; Sarzyñska, J.; Koca, J.; Orozco, M.; Cheatham III, T. E.; Kulinski, T.; Sponer, J. Single stranded loops of quadruplex DNA as key benchmark for testing nucleic acids force fields. Journal of Chemical Theory and Computation 2009, $5,2514-2530$.

(16) Orozco, M.; Noy, A.; Pérez, A. Recent advances in the study of nucleic acid flexibility by molecular dynamics. Current Opinion in Structural Biology 2008, 18, 185-193.

(17) Brackley, C. A.; Taylor, S.; Papantonis, A.; Cook, P. R.; Marenduzzo, D. Nonspecific bridging-induced attraction drives clustering of DNA-binding proteins and genome organization. Proceedings of the National Academy of Sciences 2013, 110, E3605-E3611.

(18) Michieletto, D.; Orlandini, E.; Marenduzzo, D. Polymer model with Epigenetic Recoloring Reveals a Pathway for the de novo Establishment and 3D Organization of Chromatin Domains. Physical Review X 2016, 6, 041047. 
(19) Ouldridge, T. E. Coarse-grained modelling of DNA and DNA self-assembly; Springer Science \& Business Media, 2012.

(20) Ouldridge, T. E.; Louis, A. A.; Doye, J. P. DNA nanotweezers studied with a coarsegrained model of DNA. Physical Review Letters 2010, 104, 178101.

(21) Ouldridge, T. E.; Hoare, R. L.; Louis, A. A.; Doye, J. P.; Bath, J.; Turberfield, A. J. Optimizing DNA nanotechnology through coarse-grained modeling: a two-footed DNA walker. ACS Nano 2013, 7, 2479-2490.

(22) Doye, J. P.; Ouldridge, T. E.; Louis, A. A.; Romano, F.; Šulc, P.; Matek, C.; Snodin, B. E.; Rovigatti, L.; Schreck, J. S.; Harrison, R. M.; Smith, W. P. Coarsegraining DNA for simulations of DNA nanotechnology. Physical Chemistry Chemical Physics 2013, 15, 20395-20414.

(23) Šulc, P.; Ouldridge, T. E.; Romano, F.; Doye, J. P.; Louis, A. A. Simulating a burntbridges DNA motor with a coarse-grained DNA model. Natural Computing 2014, 13, $535-547$.

(24) Leunissen, M. E.; Frenkel, D. Numerical study of DNA-functionalized microparticles and nanoparticles: Explicit pair potentials and their implications for phase behavior. The Journal of Chemical Physics 2011, 134, 084702.

(25) Angioletti-Uberti, S.; Mognetti, B. M.; Frenkel, D. Re-entrant melting as a design principle for DNA-coated colloids. Nature Materials 2012, 11, 518-522.

(26) Angioletti-Uberti, S.; Mognetti, B. M.; Frenkel, D. Theory and simulation of DNAcoated colloids: a guide for rational design. Physical Chemistry Chemical Physics 2016, 18, 6373-6393.

(27) Angioletti-Uberti, S.; Varilly, P.; Mognetti, B. M.; Frenkel, D. Mobile linkers on DNAcoated colloids: valency without patches. Physical Review Letters 2014, 113, 128303. 
(28) Reinhardt, A.; Frenkel, D. Numerical evidence for nucleated self-assembly of DNA brick structures. Physical Review Letters 2014, 112, 238103.

(29) Wayment-Steele, H. K.; Frenkel, D.; Reinhardt, A. Investigating the role of boundary bricks in DNA brick self-assembly. Soft Matter 2017, 13, 1670-1680.

(30) Fosado, Y. A. G.; Michieletto, D.; Allan, J.; Brackley, C.; Henrich, O.; Marenduzzo, D. A single nucleotide resolution model for large-scale simulations of double stranded DNA. Soft matter 2016, 12, 9458-9470.

(31) Ghobadi, A. F.; Jayaraman, A. Effect of backbone chemistry on hybridization thermodynamics of oligonucleic acids: a coarse-grained molecular dynamics simulation study. Soft Matter 2016, 12, 2276-2287.

(32) Condon, J. E.; Jayaraman, A. Effect of oligonucleic acid (ONA) backbone features on assembly of ONA-star polymer conjugates: a coarse-grained molecular simulation study. Soft Matter 2017, 13, 6770-6783.

(33) Starr, F. W.; Sciortino, F. Model for assembly and gelation of four-armed DNA dendrimers. Journal of Physics: Condensed Matter 2006, 18, L347.

(34) Kremer, K.; Grest, G. S. Dynamics of entangled linear polymer melts: A moleculardynamics simulation. The Journal of Chemical Physics 1990, 92, 5057-5086.

(35) Plimpton, S. Fast parallel algorithms for short-range molecular dynamics. Journal of Computational Physics 1995, 117, 1-19.

(36) Ness, C.; Palyulin, V. V.; Milkus, R.; Elder, R.; Sirk, T.; Zaccone, A. Nonmonotonic dependence of polymer-glass mechanical response on chain bending stiffness. Phys. Rev. E 2017, 96, 030501, DOI: 10.1103/PhysRevE.96.030501. 
(37) Milkus, R.; Ness, C.; Palyulin, V. V.; Weber, J.; Lapkin, A.; Zaccone, A. Interpretation of the Vibrational Spectra of Glassy Polymers Using Coarse-Grained Simulations. Macromolecules 2018, 51, 1559-1572, DOI: 10.1021/acs.macromol.7b02352.

(38) Di Michele, L.; Mognetti, B. M.; Yanagishima, T.; Varilly, P.; Ruff, Z.; Frenkel, D.; Eiser, E. Effect of inert tails on the thermodynamics of DNA hybridization. Journal of the American Chemical Society 2014, 136, 6538-6541.

(39) Geerts, N.; Eiser, E. DNA-functionalized colloids: Physical properties and applications. Soft Matter 2010, 6, 4647-4660.

(40) SantaLucia, J. A unified view of polymer, dumbbell, and oligonucleotide DNA nearestneighbor thermodynamics. Proceedings of the National Academy of Sciences 1998, 95, $1460-1465$.

(41) Müller, E. A.; Gubbins, K. E. Molecular-based equations of state for associating fluids: A review of SAFT and related approaches. Industrial \& Engineering Chemistry Research 2001, 40, 2193-2211.

(42) Evans, D. J.; Morriss, G. Nonlinear-response theory for steady planar Couette flow. Physical Review A 1984, 30, 1528.

(43) Daivis, P. J.; Todd, B. A simple, direct derivation and proof of the validity of the SLLOD equations of motion for generalized homogeneous flows. The Journal of Chemical Physics 2006, 124, 194103. 


\section{7 for Table of Contents use only}

TITLE: Structural and linear elastic properties of DNA hydrogels by coarse-grained simulation

AUTHORS: Zhongyang Xing, Christopher Ness, Daan Frenkel and Erika Eiser.
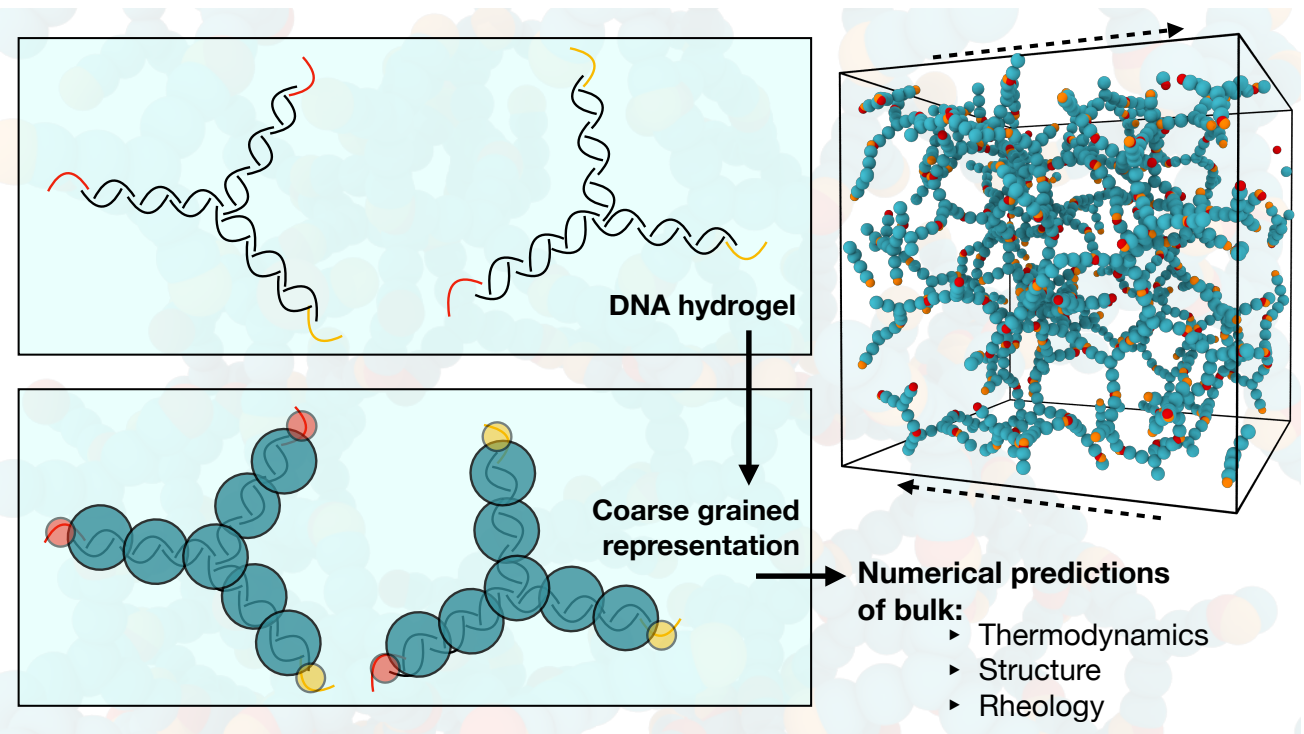

Figure 6: for Table of Contents use only 